http://www.inass.org/

\title{
Research on Intelligent Test System for Solenoid Valve
}

\author{
Yang Jiang*, Hongyi Liu, Zhong Luo, Xi Chen \\ School of Mechanical Engineering \& Automation, Northeastern University, Shenyang 110004, P.R.China \\ *Corresponding author’s E-mail address: beyond821015@163.com
}

\begin{abstract}
The solenoid valve is a key control element of hydraulic system which plays a decisive role in the reliability and the level of automation of the equipment. An intelligentized, comprehensive and high efficient test system of solenoid valve is designed in this paper. The mathematical model of the test system is built after ignoring the unimportant factors, and the visual modeling is built with the AMESim software. Fuzzy turning PID controller which changes the three parameters of PID controller by the error and error's varieties of systems is used to control the system pressure; the performance of system is obtained by simulating with combining MATLAB/Simulink and AMESim. 5Bar, 15Bar and 25Bar are given as the target value, and the best controlling strategy after the analysis and comparison is got to improve the test efficiency and test results of solenoid valve.
\end{abstract}

Keywords: solenoid valve; pressure control; AMESim; intelligent-test system; LabVIEW; fuzzy PID control

\section{Introduction}

The solenoid valve is a key control element of hydraulic system which plays a decisive role in the reliability and the level of automation of the equipment. So the reliability and the performance test of solenoid valve are of great importance, therefore solenoid valve test system is designed. This system can test their action performance, sealing performance, leakage and life. A key requirement in all the tests is to keep the given pressure for a certain time, so the research is carried on this problem.

With the rapid development of modern industry and computer technology, the development direction of test goes beyond traditional manual test method, and turns on to the concentrations on the intelligent automation test method by computer, test control, and virtual instrument technology[1]. Because automatic control of many current test experiments for solenoid valve are not achieved yet, in order to meet the demand of the development of production, it is necessary to design an electromechanical integrated equipment-solenoid valve intelligent test system, which should be with the quality of high-performance, practicality, good expansibility, energy saving and environmental protection.

\section{Design of test system scheme}

Solenoid valve test system for oil medium is designed by the following scheme, which strictly abides by national norms and industry standards with fully considering the actual situation and demand, as shown in Figure 1.

From Figure 1 two pipelines can be seen in this system, which includes $50 \mathrm{~mm}$ and $100 \mathrm{~mm}$, and motion test, seal test, leak test and life test can be realized respectively by each pipeline. The solenoid valve can be worked precisely and reliably before use by these tests.

The test principles of the system are as follows: the pipeline is chosen according to the diameter of tested-valve, and then to open electric globe valve and electric regulating valve after tested-valve is clamped by clamp cylinder; pipeline will be filled with oil by starting pumps, and the pressure is measured through data acquisition card. Once the 


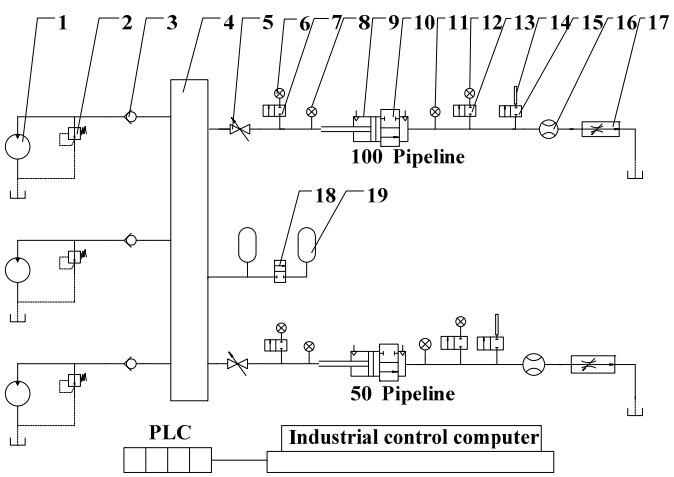

1- Oil pump;2-Overflow valve;3-Check valve;4-Oil allocation cylinder;5-Electric globe valve; 6,12-Small pressure sensor;7,13,15,18-Switch valve;8,11-Big pressure sensor;9-Clamp cylinder;10-Tested-vavle;14- Leakage tube;16-

Flow sensor;17- Electric regulating valve;19- Accumulator

Figure 1 Structure scheme of solenoid valve test system for oil medium

pressure meets test demand, experiment will be staring. When the experiment has been completed, tested-valve and the electric regulating valve will be opened with all pumps closing, and then oil will be returned to the oil tank naturally. The tested-valve can be taken down when the flux acquired by data acquisition card from the flow sensor is zero. This test is completed, then to start the next one.

\section{Design of control scheme}

\subsection{Design of general control scheme of system}

The general control scheme of system is shown in Figure 2. Master computer is industrial control computer based on LabVIEW software with data acquisition card inside, which will complete planning, data acquisition of the whole system, as well as communications with PLC; PLC (Programmable Logic Controller) is used as the slave computer, solenoid valve action sequence control in each experiment can be completed by it. NI DataSocket technology and OMRON's OPC-SERVER server has been applied between master computer and slave computer, and RS-232C communication mode is applied to implement the serial communication of them.

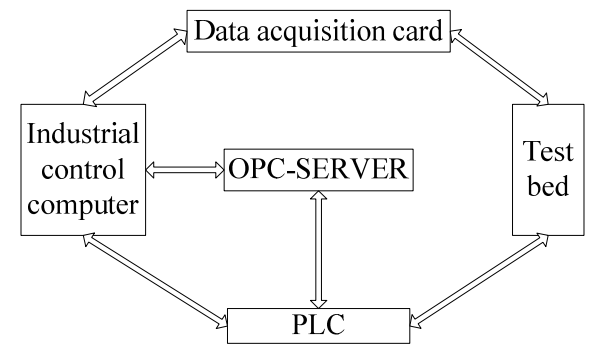

Figure 2 General controlling frame of system

\subsection{Design of pressure control scheme of system}

A certain pressure is required to achieve and maintain in the seal and leak test in order to guarantee the process of the experiment normally. So the pressure in the pipeline can be controlled by regulating the output of transducer to control pump rotate speed to achieve, closed-loop control on pressure is used in the whole system, as shown in Figure 3.

Its control process is as follows: system pressure is tested real-time by pressure sensor, and is transmitted to industrial control computer by data acquisition card; Data are processed by industrial control computer according to predetermined control strategy, and the processing results are transmitted to the PLC by OPC; the signals will be sent to the hydraulic pump and electric regulating valve, as well as each switch valve after they have been read and analyzed by PLC.

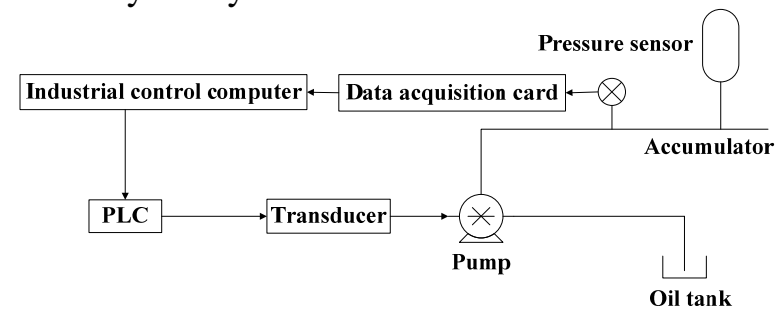

Figure 3 The pressure control of test system

\section{The mathematical model establishment of test system}

The pressure part of this solenoid valve test system includes quite many hydraulic elements, and mathematical model of each part is also unusual complex. A method of white-black-gray box combining hydraulic system sub-model is used to establish the system model.

\subsection{Commonly used hydraulic system sub-model}

\subsubsection{Oil pump}

A fixed displacement pump is as shown in figure 4, and its sub-model can be expressed as:

$$
q_{1}=q_{0}-G_{1}\left(p_{1}-p_{0}\right)-\frac{V_{1}}{K} p_{1}
$$

where $q_{0}$ and $q_{1}$ denote theoretical flow and actual flow of oil pump, respectively, $G_{1}$ denotes flow conductance of oil pump, $V_{1}$ denotes pump outlet volume, and $K$ denotes hydraulic oil bulk modulus. 


\subsubsection{Relief valve}

The structure of direct operated relief valve is as shown in Figure 5.

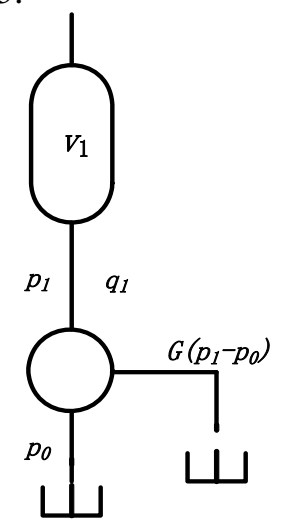

Figure 4 The system of a fixed displacement pump

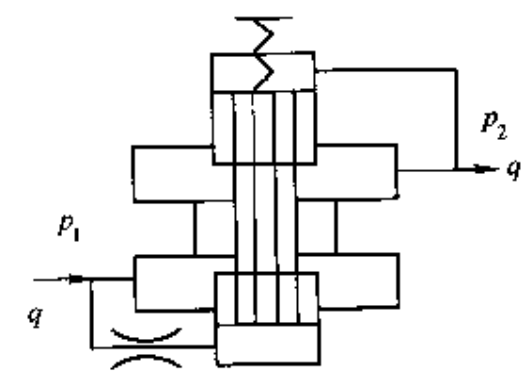

Figure 5 The structure of direct operated relief valve

The valve core motion equation is shown as following equation:

$m_{r} \ddot{x}_{v}=\left(p_{1}-p_{2}\right) A_{r}-K_{s}\left(x_{c}+x_{v}\right)-\left(K_{v}+K_{f}\right)$

where $m_{r}$ denotes quality and displacement of valve core, $p_{1}$ and $p_{2}$ denote inlet and outlet oil pre- ssure, respectively, $K_{s}$ and $x_{c}$ denote spring stiffness and pre-compressing value, respectively, $K_{v}$ and $K_{f}$ denote transient flow force and elastic damping coefficient, respectively, $A_{r}$ denotes fluid operation area.

In hydraulic large-scale system, if neglects the dynamic characteristic of relief valve's opening, its simplified model can be expressed as

$$
q=G_{2}\left(p_{1}-p_{r}\right)
$$

where $G_{2}$ denotes flow conductance of relief valve, and $p_{r}$ denotes adjustable pressure of relief valve.

\subsubsection{Solenoid directional control valve}

When solenoid directional control valve is electrifying, the valve core motion equation is:

$$
F_{e}=m \ddot{x}=\left(B_{v}-B_{f}\right) \dot{x}+\left(K_{V}+K_{s}\right) x+R_{m}
$$

where $F_{e}$ denotes electromagnetic force, $m$ and $x$ denote quality and displacement of valve core, respectively, $B_{v}$ and $B_{f}$ denote transient flow force and viscous damping coefficient, respectively, and $R_{m}$ denotes coulomb frictional resistance.

After solenoid valve commutation, flow equation which flows through solenoid valve is as following equation.

$$
q=C_{d} A \sqrt{2\left(p_{1}-p_{2}\right) / \rho}=C \sqrt{p_{1}-p_{2}}
$$

where $C_{d}$ and $A$ denote valve port discharge coefficient and valve port area, respectively, $\rho$ denotes fluid density, $p_{1}$ and $p_{2}$ denote inlet and outlet fluid pressure of solenoid valve, respectively, and $K_{v}$ denotes aggregate discharge coefficient of solenoid valve.

The response speed of solenoid directional control valve is quick, so discharge characteristic is only considered when in consideration dynamic property of large-scale system. Its mathematical model is:

$$
q=C_{d} A \sqrt{2\left(p_{1}-p_{2}\right) / \rho}=K_{V} \sqrt{\left(p_{1}-p_{2}\right) / \rho}
$$

\subsubsection{Accumulator}

Accumulator model under the condition of isothermal process is different from adiabatic process, and dynamic model under the condition of isothermal process is researched by this article.

The ideal gas equation is

$$
P V=M R T
$$

When aeration finished, the gas whose quality is $\mathrm{M}$ had entered into the accumulator, if the variation of accumulator pressure was slow, then temperature $\mathrm{T}$ can be regarded as a constant, so was R. This means working under the condition of isothermal process.

$$
V_{a}=\frac{M R T}{p_{a}}=V_{a, 0}-\int_{0}^{t} Q_{f} d t
$$

where $V_{a}$ and $p_{a}$ denote volume and pressure of compressed gas, respectively, $V_{a, 0}$ denotes gas volume after finishing aeration, $Q_{f}$ denotes flux which flows to accumulator, and $M R T$ is gas con- stant.

$$
\begin{aligned}
p_{f} & =p_{a} \\
p_{f} & =\frac{M R T}{V_{a}}=\frac{M R T}{V_{a, 0}-V_{f}}
\end{aligned}
$$


where $V_{f}$ denotes oil bulk volume which flows in the accumulator.

\subsubsection{Check valve}

The motion equation of straight-through check valve is:

$$
m \ddot{x}=\left(p_{1}-p_{2}\right) A-K_{s} x-F_{f}
$$

where $F_{f}$ denotes pre-tightening force.

$$
q=\frac{1}{R}\left(p_{1}-p_{2}\right)=G\left(p_{1}-p_{2}\right)
$$

where $R$ denotes liquid resistance, and $G$ denotes flow conductance.

\subsubsection{Electric regulating valve}

Linear regulating valve:

$$
q=\left(k l+k_{0}\right) \sqrt{\left(p_{1}-p_{2}\right) / \rho}
$$

Equal jigging percentage regulating valve:

$$
q=e^{k l+k_{0}} \sqrt{\left(p_{1}-p_{2}\right) / \rho}
$$

where $k$ denotes regulating valve constant $\left(\mathrm{m}^{2} / \mathrm{s}\right)$, $l$ denotes relative opening degree of regulating valve, and $k_{0}$ denotes constant $\left(\mathrm{m}^{2} / \mathrm{s}\right)$.

\subsection{Pressure control system mathematical model establishment}

This test system does not allow two pipelines simultaneously to work, only needs to establish mathematics model to a pipeline. Thus, the model of 100 pipeline is established as shown in Figure 6.

In the pipeline, the relief valve plays the protective function, and does not have the overflow in the normal condition. Therefore, the overflow question is not considered normally. Moreover, the pressure loss of filter is small to the system influence, therefore it can be ignored. So the system model except for these parts (subsystem) is as following equations.

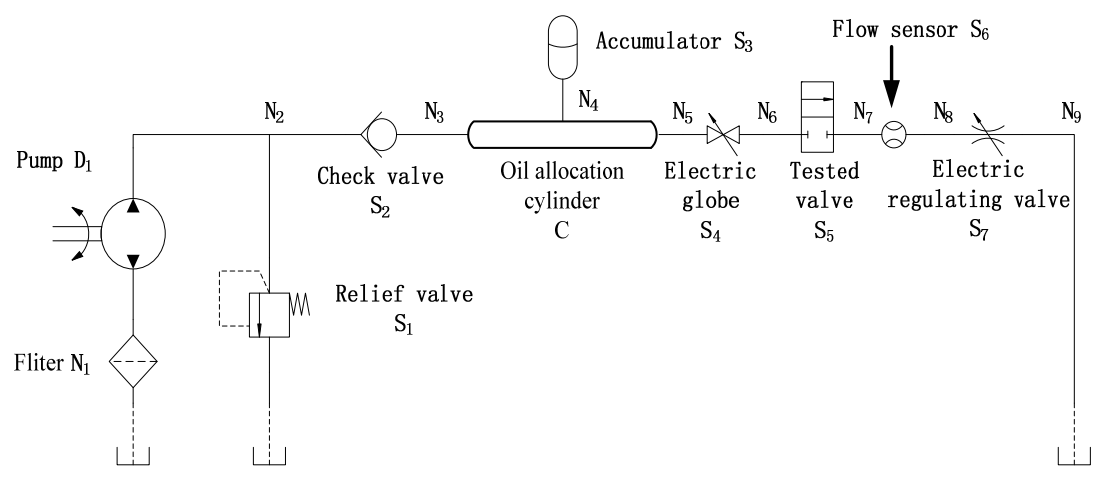

Figure 6 The system of 100 pipelines

$$
\begin{aligned}
& \mathrm{D}_{1}: q_{2}=q_{1}-G_{1}\left(p_{2}-p_{1}\right)-\frac{V_{1}}{K} p_{2} \\
& \mathrm{~S}_{2}: q_{3}=\frac{1}{R_{2}}\left(p_{3}-p_{2}\right)=G_{2}\left(p_{3}-p_{2}\right) \\
& \mathrm{C}: \quad q_{3}-q_{4}-q_{5}=C_{f} \frac{d p_{c}}{d t} \\
& \mathrm{~S}_{3}: p_{f}=p_{c}=\frac{M R T}{V_{a, 0}-V_{f}}=\frac{M R T}{V_{a, 0}-\int_{0}^{t} q_{4} d t} \\
& \mathrm{~S}_{4}: q_{6}=K_{V} \sqrt{\left(p_{6}-p_{5}\right) / \rho} \\
& \mathrm{S}_{5}: q_{7}=K_{V} \sqrt{\left(p_{7}-p_{6}\right) / \rho} \\
& \mathrm{S}_{6}: \quad q_{8}=\frac{p_{8}-p_{7}}{R} \\
& \mathrm{~S}_{7}: q_{9}=\left(k l+k_{0}\right) \sqrt{\left(p_{9}-p_{8}\right) / \rho}
\end{aligned}
$$

Liquid resistance and flow conductance are ignoring of mostly pipeline, so

$$
\begin{aligned}
& q_{2}=q_{3} \\
& q_{5}=q_{6}=q_{7}=q_{8}=q_{9}
\end{aligned}
$$

After establishing above various modules' mathematical models, they can be realized in MATLAB/Simulink according to the modern control theory method, then the recombination pressure control strategy carries on the simulation.

\section{The AMESim simulation model establish- ment of test system}

The system model is established by hydraulic simulation software AMESim. This software contains IMAGINE skill, and can provide the interactive ability for the engineering design. It is a hydrodynamic (fluid and gas), mechanical, thermal fluid and control system which provides a consummation, superior simulation environment and the nimble solution, like in braking system, power drive, mechanical and electrical system's application. The 
friendly operation interface faces to practical application which can satisfy demands of users.

If simulation model is established to the whole system with large amount of computation, simulation results will be affected unexpectedly which will not benefit the analysis of problem. Therefore, to simplify the simulation process, and focus on the more important factors, the model is simplified. The simplified AMESim model is shown in Figure 7.

A "PRESSURE" box can be seen from Figure 7, which is the connecting box of AMESim and MATLAB / Simulink.

(C)

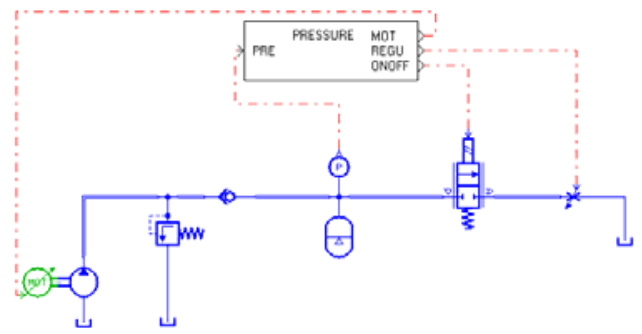

Figure 7 The simplified AMESim model of test system

\section{Design of fuzzy PID controller for pressure and simulation analysis}

It is difficult to establish precise mathematical model on this test system, because many components are contained in it. The system is timedependent, thermosensitive and nonlinear, so gain self-tuning fuzzy PID control is used for the pressure control of system.

\subsection{Frame of gain self-tuning fuzzy PID controller}

Gain self-tuning fuzzy PID control system is a kind of adaptive system, which is to regulate PID parameters real-time according to fuzzy control rules. It includes a linear controller PID controller and, and PID parameters can be regulated based on the expert knowledge of the experience in the fuzzy inference engine. The principle of gain self-tuning fuzzy PID control is shown in Figure 8.

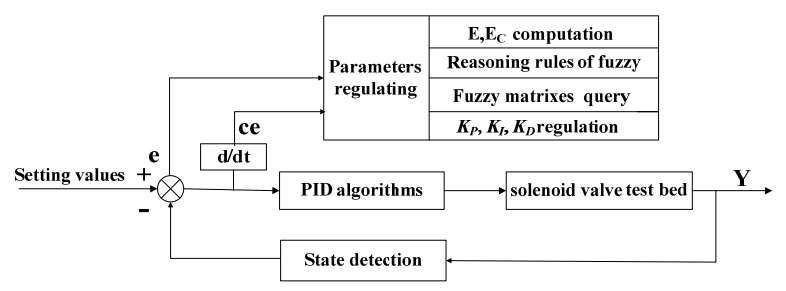

Figure 8 Principle chart of gain self-tuning fuzzy PID controller of the test system

\subsection{Establishment of fuzzy rules tables of PID parameters}

An analytic expression can be used to sum up the relationship of various PID parameters, system error and error rate of change according to the methods that analytic expression describes control rules, which use an adjusting factor $\alpha$ to express different weighted degree on the system error and error rate of change by PID parameters. The universe of discourse of $\mathrm{K}_{\mathrm{P}}, \mathrm{K}_{\mathrm{I}}, \mathrm{K}_{\mathrm{D}}$ parameters, system error and error rate of change are chosen, as well as each value in it. The initial values of $K_{P}, K_{I}$, $\mathrm{K}_{\mathrm{D}}$ can be obtained by chosen of different adjusting factor $\alpha$ in different stages of system based on the relationship analysis of $\mathrm{K}_{\mathrm{P}}, \mathrm{K}_{\mathrm{I}}, \mathrm{K}_{\mathrm{D}}$ parameters, system error and error rate of change. $K_{P}, K_{I}, K_{D}$ values can be determined finally according to parameters setting and the fuzzy decision by maximum membership degree. Fuzzy rules are as shown in Tables 1 to 3 .

Table 1 Fuzzy control value of $K_{\mathrm{P}}$

\begin{tabular}{|c|c|c|c|c|c|c|c|c|}
\hline \multicolumn{2}{|c|}{$K_{\mathbf{P}}$} & \multicolumn{7}{|c|}{$\mathbf{E}$} \\
\cline { 2 - 9 } & -3 & -2 & -1 & 0 & 1 & 2 & 3 \\
\hline \multirow{4}{*}{} & -3 & 3.0 & 3.0 & 3.0 & 3.0 & 2.0 & 1.0 & 0 \\
\cline { 2 - 9 } $\mathbf{E}_{\mathbf{C}}$ & -2 & 3.0 & 3.0 & 3.0 & 3.0 & 1.5 & 0 & 0 \\
\cline { 2 - 9 } & -1 & 2.0 & 2.0 & 2.0 & 2.0 & 1.0 & 0 & -1.0 \\
\cline { 2 - 9 } & 0 & 2.0 & 1.0 & 1.0 & 0 & -1.0 & -1.0 & -1.0 \\
\cline { 2 - 9 } & 1 & 1.0 & 0 & -1.0 & -2.0 & -2.0 & -2.0 & -2.0 \\
\cline { 2 - 9 } & 2 & 0 & 0 & -2.0 & -3.0 & -3.0 & -3.0 & -3.0 \\
\cline { 2 - 8 } & 3 & 0 & -1.0 & -2.0 & -3.0 & -3.0 & -3.0 & -3.0 \\
\hline
\end{tabular}

Table 2 Fuzzy control value of $\mathrm{K}_{\mathrm{I}}$

\begin{tabular}{|c|c|c|c|c|c|c|c|c|}
\hline \multicolumn{2}{|c|}{$\mathbf{K}_{\mathbf{I}}$} & \multicolumn{7}{|c|}{$\mathbf{E}$} \\
\cline { 2 - 9 } & -3 & -2 & -1 & 0 & 1 & 2 & 3 \\
\hline \multirow{4}{*}{$\mathbf{E}_{\mathbf{C}}$} & -3 & 1.0 & -3.0 & -3.0 & -1.0 & -3.0 & -3.0 & 1.0 \\
\cline { 2 - 9 } & -2 & 1.0 & -3.0 & -3.0 & -1.0 & -3.0 & -3.0 & 1.0 \\
\cline { 2 - 9 } & -1 & 0 & -2.0 & -2.0 & -1.0. & -2.0 & -2.0 & 0 \\
\cline { 2 - 9 } & 0 & 0 & -1.0 & -1.0 & -1.0 & -1.0 & -1.0 & 0 \\
\cline { 2 - 9 } & 1 & 0 & 2.0 & 1.0 & 0 & 1.0 & 2.0 & 0 \\
\cline { 2 - 9 } & 2 & 1.0 & 3.0 & 1.0 & 1.0 & 3.0 & 3.0 & 1.0 \\
\cline { 2 - 9 } & 3 & 1.0 & 3.0 & 2.0 & 2.0 & 3.0 & 3.0 & 1.0 \\
\hline
\end{tabular}

Table 3 Fuzzy control value of $K_{D}$

\begin{tabular}{|c|c|c|c|c|c|c|c|c|}
\hline \multicolumn{2}{|c|}{$\mathbf{K}_{\mathbf{D}}$} & \multicolumn{7}{|c|}{$\mathbf{E}$} \\
\cline { 2 - 9 } & -3 & -2 & -1 & 0 & 1 & 2 & 3 \\
\hline \multirow{4}{*}{$\mathbf{E}_{\mathbf{C}}$} & -3 & -3.0 & -2.0 & -2.0 & -1.0 & -1.0 & 0 & 0 \\
\cline { 2 - 9 } & -2 & -3.0 & -2.0 & -2.0 & -1.0 & 0 & 0 & 0 \\
\cline { 2 - 9 } & -1 & -2.0 & -2.0 & -1.0 & 0 & 0 & 0 & -1.0 \\
\cline { 2 - 9 } & 0 & 1.0 & 1.0 & 0 & 0 & 0 & -1.0 & -1.0 \\
\cline { 2 - 9 } & 1 & 1.0 & 0 & 0 & 0 & 1.0 & 2.0 & 2.0 \\
\cline { 2 - 9 } & 2 & 0 & 0 & 0 & 1.0 & 1.0 & 2.0 & 2.0 \\
\cline { 2 - 9 } & 3 & 0 & 0 & 1.0 & 1.0 & 2.0 & 3.0 & 3.0 \\
\hline
\end{tabular}




\subsection{Simulation experiment and results analysis}

Firstly, to fuzz $\Delta \mathrm{e}, \Delta \mathrm{e}$ and $\mathrm{K}_{\mathrm{P}}, \mathrm{K}_{\mathrm{I}}, \mathrm{K}_{\mathrm{D}}$ variables, and determine the membership of their fuzzy subsets. Secondly, to determine the universe of parameters: $K_{P}, E, E_{C}=(-6,-5,-4,-3,-2,-1,0,1,2,3,4,5,6), K_{I}$, $K_{D}=(-3,-2,-1,0,1,2,3)$, as well as their fuzzy subsets: $\mathrm{E}, \mathrm{E}_{\mathrm{C}}=(\mathrm{NB}, \mathrm{NM}, \mathrm{NS}, \mathrm{O}, \mathrm{PS}, \mathrm{PM}, \mathrm{PB}), \mathrm{K}_{\mathrm{P}}$, $\mathrm{K}_{\mathrm{I}}, \mathrm{K}_{\mathrm{D}}=(\mathrm{NB}, \mathrm{NM}, \mathrm{NS}, \mathrm{O}, \mathrm{PS}, \mathrm{PM}, \mathrm{PB})$. Lastly, to fuzz $E, E_{C}, K_{P}, K_{I}, K_{D}$ and membership function are as shown in Figures 9 to 11.

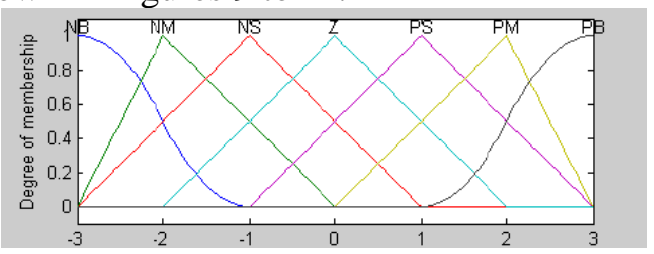

Figure 9 Membership function curve of E, EC and $\mathrm{K}_{\mathrm{D}}$

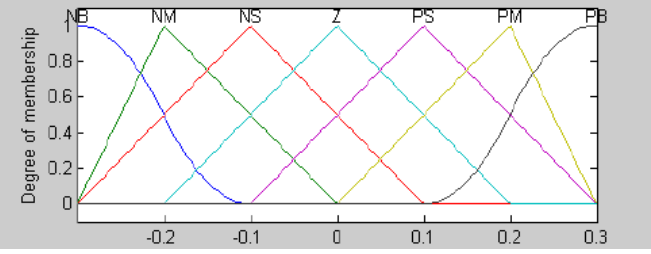

Figure 10 Membership function curve of $\mathrm{K}_{\mathrm{P}}$

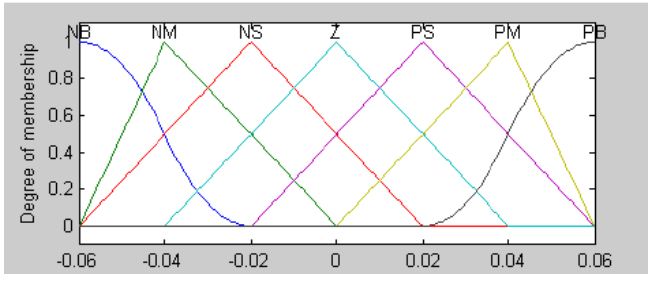

Figure 11 Membership function curve of $\mathrm{K}_{\mathrm{I}}$

To start simulation after the corresponding parameters which are stetted properly based on MATLAB / Simulink. Target values of the system are respectively 5Bar, 15Bar, 25Bar step signals, and the simulation curve is shown in Figure 12.

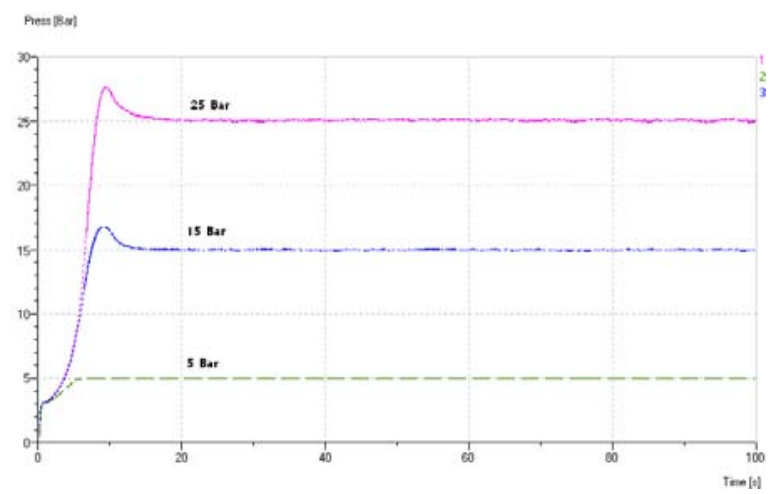

Figure 12 Simulation result of gain self-tuning fuzzy PID

The $\mathrm{K}_{\mathrm{P}}, \mathrm{K}_{\mathrm{I}}, \mathrm{K}_{\mathrm{D}}$ parameters of model is tested in experiments, and its self-tuning curves are shown in Figures 13 to 15.

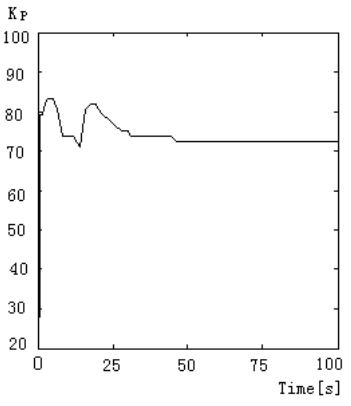

Figure 13 The self-turning process of $K_{P}$ for step response

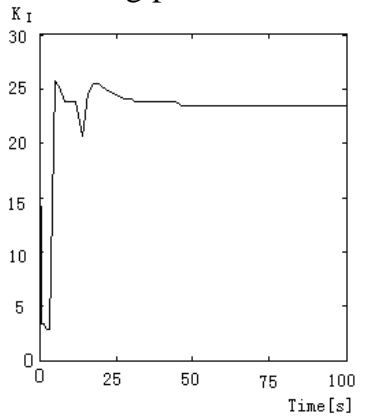

Figure 14 The self-turning process of $\mathrm{K}_{\mathrm{I}}$ for step response

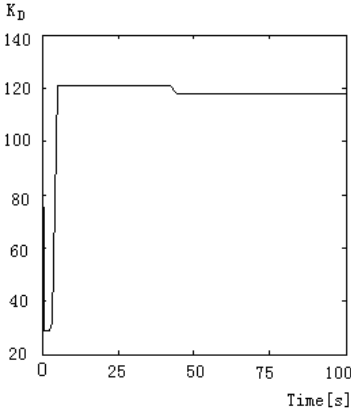

Figure 15 The self-turning process of $\mathrm{K}_{\mathrm{D}}$ for step response

The response overshoot is small by using the gain self-tuning fuzzy PID controller from observing the simulation results. The maximum overshoot of target value for the $15 \mathrm{Bar}$ is $13 \%$, and is $12 \%$ for the 25Bar, and they can both meet the system requirements. The response speed is quick, and the biggest adjustment time is: $\mathrm{t}=12 \mathrm{~s}$. In addition, the steady-state error of response curve is small, too.

\section{Conclusion}

(1) Design of test system scheme for the oil medium solenoid valve that runs through field debugging has been completed. It is proved to be safe and reliable, and has a bright application prospect.

(2) The intelligent control of system pressure and automatic control purposes of system have been completed by comprehensive utilization PLC technology, data acquisition technology based on LabVIEW and OPC-SERVER serial communication 
technologies.

(3) The solenoid valve test system overall construction and the pressure control system have been studied. The system pressure mathematical model and the AMESim simulation model have been established, and very good expression is delivered to this system.

(4) The gain of self-adjusting fuzzy PID control methods is used on the pressure of system. After the simulation test, the results show that each performance index of the system with a good selfadjusting function, when interfered, can reach the design requirements with good results.

\section{References}

[1] M.M. Ardehali, M. Saboori, M. Teshnelab, "Numerical simulation and analysis of fuzzy PID and PSD control methodologies as dynamic energy efficiency measures", Energy Conversion and Mana- gement, 2004, 45: 1981-1992.

[2] C.I. Siettos, G.V. Bafas, "Semiglobal stabilization of nonlinear systems using fuzzy control and singular perturbation methods”, Fuzzy Sets and Systems, 2004, 129: 275-294.

[3] F.H.F. Leung, H.K. Lam, P.K.S. Tam, "Design of fuzzy controllers for uncertain nonlinear systems using stability and robustness analyses”, Systems Control Lett, 1998, 35: 237-243.

[4] H.Ying. Analytical Relationship between the Fuzzy PID controllers and the linear PID controller, Technical Reprot, Department of Biomedical Engineering, The University of Albama at Birmingham, December 8, 1987.

[5] A.Ramirez-Serrano, S.C. Zhu, S.K.H. Chan, S.S.W. Chan, M. Ficocelli and B. Benhabib, "A hybrid PC/PLC architecture for manufacturing-system control-theory and implementation”, Journal of Intelligent Manufacturing, 2002, 13:261-281.

[6] James Carvajal, Guanrong Chen, Haluk Ogmen, "Fuzzy PID controller: Design, performance evaluation, and stability analysis”, Information Sciences 2000, 123: 249-270.

[7] Javier Aracil, Francisco Gordillo, "Describing function method for stability analysis of PD and PI fuzzy controllers”, Fuzzy Sets and Systems, 2004 143: 233-249.

[8] Dragan D. Kukolj, Slobodan B.Kuzmanovic, Emil Levi, "Design of a PID-like compound fuzzy logic controller", Engineering Applications of Artificial Intelligence, 2001, 14: 785-803 